\title{
Kernos
}

Revue internationale et pluridisciplinaire de religion grecque antique

16 | 2003

Varia

\section{«Les mots qui voient ». Du tragique dans le Prométhée enchaîné}

\section{Ioanna Papadopoulou-Belmehdi}

\section{(2) OpenEdition \\ Journals}

Édition électronique

URL : http://journals.openedition.org/kernos/810

DOI : $10.4000 /$ kernos.810

ISSN : 2034-7871

\section{Éditeur}

Centre international d'étude de la religion grecque antique

\section{Édition imprimée}

Date de publication : 1 janvier 2003

Pagination : 43-57

ISSN : 0776-3824

\section{Référence électronique}

Ioanna Papadopoulou-Belmehdi, « «Les mots qui voient ». Du tragique dans le Prométhée enchainné », Kernos [En ligne], 16 | 2003, mis en ligne le 14 avril 2011, consulté le 30 avril 2019. URL : http:// journals.openedition.org/kernos/810; DOI : 10.4000/kernos.810 


\title{
«Les mots quii voient » Du tragique dans le Promethée enchâ̂né
}

\author{
It is this relation, witb its paradox of fidelity \\ to the source and autonomous counter-statement, \\ which constitutes the vitality of interpretation. \\ On this rare level one can, without irony, compare \\ the bermeneutic with the poetic act ${ }^{1}$. \\ À la mémoire de Nicole Loraux
}

\section{Opsis contre lexis?}

Dans un article remarquable, qui s'intitule « Les mots qui voient », Nicole Loraux s'interroge sur le mot ó « comme remarquable présence du voir dans le dire tragique $»^{2}$.

Dans sa division de la production tragique en quatre espèces, Aristote classe le

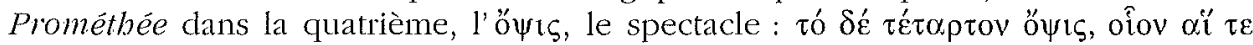

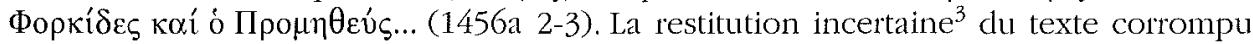
en $56 a 3$ est commentée de manière convaincante, me semble-t-il, par R. Dupont-Roc

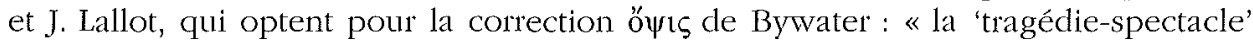
apparaîtrait donc comme un type extrême dans lequel l'hypertrophie du spectaculaire inhérent à l'effet violent peut aller de pair avec une extrême simplicité de l'histoire »4

Au-delà du problème spécifique à ce passage lacunaire, il est intéressant de constater à quel point ces deux catégories-« repoussoirs» de la théorie aristotélicienne du tragique informent une certaine attitude érudite à l'égard du Prométhée enchâné. L'analogie est pesante : d'une part elle fait oublier l'évidence d'une antériorité chronologique, d'autre part elle ne pose pas l'existence d'une dimension métathéâtrale qu'Aristote pourrait solliciter beaucoup plus qu'il ne le laisse transparaître ${ }^{5}$.

1 G. Steiner, Antigones, Oxford, 1984, p. 42. Une version de ce travail a été présentée lors d'une «Journée autour de Nicole Loraux », le 17 novembre 2001 (à l'École des Hautes Études en Sciences Sociales), retravaillé à partir d'une communication présentée à Cornell University, dans le cadie du colloque Corhali («The Language of Aeschylus »), en mai 1999. Je remercie P. Pucci de l'attention qu'il a portée à ce travail.

2 Dans C. Reichlen (éd.), L'interprétation des lextes, Paris, 1989, p. 158-182.

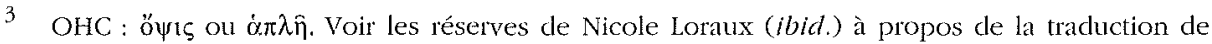
ce terme.

4 R. Dupont-Roc, J. Lallot, Aristote: La Poétique, Paris, 1980, p. 298. C'est en essayant d'établit une analogie entre l'épopée simple et la tragédie - spectacle que les auteurs procèdent à ces raisonnements qui me paraissent tout à fait fondés et qui sont inspirés de la totalité du contexte de la Poétique. Voir aussi, à propos de ów, le commentaire éclailant à 58 a 5.

5 Il s'agit d'une stratégie cohérente, voir les remarques plus générales de D. LANZA, "Redondances du mythe dans la tragédie", in C. Calame (éd.) Métamorphoses du mythe en Grèce antique, Genève, 1989, p. 141-141. Le projet aristotélicien contredit les fondements même du 
Car la question se pose d'emblée à tout lecteur de la pièce, vı le poids de préjugés déjà anciens et toujours influents : qu'y a-t-il de tragique dans le Prométbée enchaîné ? Si on utilise les catégories aristoteliciennes de la Poétique, la réponse est « rien » ou «peu de choses »; le Prométhée appartient à l'espèce-limite où l'opsis est privilégiée; en tout cas, même sí l'on admet l'option textuelle « d'une espèce tragique simple », le Promethée est un cas problématique quant à son essence tragique.

Or, c'est justement dans cette pièce qu'Eschyle donne aux mots qui désignent la terreur et la pitié le statut de thèmes-clefs, y incorporant de façon complexe l'opsis, conme je vais essayer de le démontrer dans une lecture essentiellement intratextuelle de ces termes ${ }^{6}$. C'est à dessein, me semble-t-il, que dans le Promethée le visuel règne, mais il règne surtout dans les mots. Aucune pièce d'Eschyle - ni même l'Ecdipe-Roi de Sophocle ${ }^{7}$ - ne comporte autant d'expressions relatives à la vision. Parmi toutes les tragédies existantes, le Prométbée présente la plus haute densité « des mots qui voient », plus de 75 occurences, dont la plupart sont associées à Prométhée luimême. Car, dans cette pièce, le fait accompli du malheur est avant tout appel constant à la vue, spectacle d'une horreur inévitable. J'aimerais insister sur le mot « inévitable » pour résumer une double opération : le dieu souffrant ne quitte jamais la scène et le langage érige cette présence ininterrompue en élément dramaturgique important.

C'est justement au thème de la vue que cet héros tragique hors du commun consacre ces tout premiers mots; à peine enchaîné, Prométhée convoque les éléments cosmiques majeurs ${ }^{8}$ au spectacle de sa souffrance : "Éther divin et vents à l'aile rapide, sources des rivières, sourires innombrables des vagues marines, terre mère de tous, disque du soleil qui voit tout, je vous invoque tous, regardez-moi, quelles souffrances j'endure, moi dieu, par les autres dieux » (88-92)

Dans ces premiers mots que Prométhée prononce sur scène, l'idée centrale est le dieu supplicié, désormais constitué en spectacle. Le thème d'un spectacle tout à fait inoui, jamais vu, tout à fait extravagant traverse la pièce. Or, même dans le Prométhëe enchainé, le visuel en soi est loin d'avoir le premier rôle; il ne devient élément tra-

«mécanisme” tragique eschyléen; à ce propos l'analyse de S. KLimi (Le statut du mytbe dans la Poétique d'Aristote, Bruxelles, 1997) est très éclairante. Remise en question de ce que nous entendons par «tragique», notamment à propos d'Euripide, par P. JUDET DE LA COMBE "Euripide et le tragique du non tragique", Europe (janv.-fév. 1999), p. 183-200 (ainsi que d'autres contributions dans ce même volume).

6 Je développe plus amplement certains points, notamment à partir du thème de l'éros, dans un ouvrage collectif sur le Prométbée, à paraître aux éditions Ousia, Bruxelles (en collaboration avec L. Couloubaritsis, M. Broze, F. Labrique).

7 C. Calame relève le paradoxe d'Ectipe-Roi - où le thème de la vision est central - en tant que pièce choisie par Aristote comme exemple de l'autonomie de la lexis : C. Calame, "Vision, Blindness, and Mask: The Radicalization of the Emotions in Sophocles's Oedipus Rex", in M.S. SILK (éd.), Tragedy and The Tragic, Oxford, 1996, p. 17-37 avec la réponse de R. Buxton dans le même volume. À propos de la vision en tant que thème et réflexion métathéâtrale dans le Prométbée, voir T. TARkow, "Sight and Seeing in the Prometheus Bound, Eranos 84 (1986), p. 87-99. Pour une brève mise en garde contre la dévalorisation de l'opsis dans la Poétique et contre l'influence de ce jugement arbitraire sur l'érudition, voir O. TAPlin, Stagecraft of Aescbylus, Oxford, 1977, Appendix F.

8 Commentaire de ce passage "empédocléen " dans C.J. Heringron, "A study in the Prometheia. Part I, The elements of the Trilogy", Phoenix 17 (1963), p. 180-197.

9 J'ai utilisé les traductions de la Collection des Universités de France, parfois modifiées. J'ai également consulté avec profit la traduction de $M$. Gondicas et P. Judet de La Combe, aux éditions Comp'Act, Chambéry, 1996. 
gique que dans son interaction avec les $\operatorname{mots}^{10}$, qui ont, eux, une certaine autonomie par rapport à l'opsis. C'est en effet avec des indications on ne peut plus minutieuses que le supplice sera en même temps donné à voir et raconté avec moultes détails " techniques », de façon qu'on puisse le voir dans les mots. Car le Prométhée ne déroge pas à la règle tragique selon laquelle, comme le dit Nicole Loraux, "les mots assument l'essentiel », ils assument la mort, les blessures, qui n'ont pas « d'autre lieu que l'énoncé ».

La parole du Titan occupe tout : du fait de sa posture, l'action ne peut être que discours; la praxis à proprement parler dans la pièce se dresse comme son extrême opposé : à la pure parole qu'est Prométhée s'oppose la figure silencieuse mais toutepuissante de Zeus, « représenté » par un seul aspect de sa configuration : Kratos, Bia. Théologique et visuel sont ici solidaires pour mener à bien le projet : l'auteur confie la «mise en scène » terrifiante à Zeus, c'est l'aspect visible de sa volonté qui occupe la scène, le logos se livrant un duel désespéré avec le Pouvoir et la Violence du dieu suprême.

Corps suspendu aux rochers d'une montagne visible de loin ( Prométhée oppose son supplice au thème traditionnel de la précipitation des Titans dans le Tartare; là aussi, c'est le fait d'être vu qui est mis en avant et non la douleur : «Ah, que ne m'a-t-il précipité sous la terre, plus bas que l'Hadès, hospitalier aux morts, jusqu'à l'impénetrable tartare, et mis au contact farouche des liens qu'on ne clélie pas, afin que nul dieu, nul être n'y trouvât à se réjouir, tandis que maintenant, jouet des airs, misérable, je souffre pour la joie de mes ennemis » (152-156).

C'est par les mots $\alpha i \kappa i \alpha, \alpha i \kappa i \zeta o \mu \alpha 1, \alpha i \kappa i \sigma \mu \alpha$ que le supplice est désigné, de façon récurrente. Le choix de ces mots ${ }^{11}$ évoque le langage épique, en privilégiant le registre de la non-semblance, de la blessure déformante, du traitement corporel dégradant.

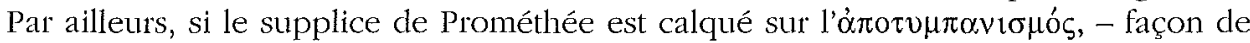
mettre à mort atroce, réservée aux traîtres et aux " malfaiteurs » ${ }^{12}-$, l'infâmie de l'exposition à la vue de tous démeure également un élément central. Néanmoins, pour rester dans les enjeux du tragique, - même dans la plus intense humiliation, Prométhée reste un dieu, grâce au langage : l'utilisation répétée du mot $\alpha i k i \alpha$, au sein d'un registre d'《 auto-affirmation $»^{13}$, renvoie aux valeurs épiques, notamment au corps héroïque abattu et exposé, possession impie de l'ennemi, qui en use à sa guise et qui le déforme, degré ultime de l'horreur, pire que la mort.

10 TAPLin, o.c. (n. 7), p. 39-49.

11 Voir M. Griffith, Aescbylus, Prometbeus Bound, Cambridge, 1983, au vers 93; A. Podlecki, The Political Background of Aeschylean Tragedy, Michigan, 1966, p. 118-119. Si Prométhée ne peut pas mourir, Zeus use de son corps avec la même liberté qu'un guerrier vainqeur peut traiter le corps de l'ennemi qu'il a tué (voir à ce propos l'article de J.-P. Vernanr, "La belle mort et le cadavre outragé", in G. GNoli et J.-P. Vernant, La mort, les morts dans les sociétés anciennes, Cambridge 1982, p. 45-76. Sur l'homérisme d'Eschyle, voir P. JUder de La Combe, "Sur la reprise d'Homère par Eschyle", Lexis 13 (1995), p. 129-144, qui traite la question d'un autre point de vue que le Aeschylus Homericus de Sideras.

12 L. Gernet, Antbropologie de la Grèce antique, Paris, 1976, p. 303-329, avec les mises au point de E. Cantarella, Les peines de mont en Grèce et à Rome, Paris, 2000, p. 30-40. Voir aussi le commentaire intéressant de F. Létoublon, "Les paradoxes du Prométhée", Sileno (janv,-déc. 1986), p. $33-34$.

13 Le terme renvoie à l'analyse de K. Reinhardt, Eschyle, Euripide, Paris, 1972, p. 52-60. 
Mais Prométhée ne peut pas mourir même si son intégrité corporelle est atteinte. La notion de la non-semblance est à verser au vocabulaire de la terreur et renvoie sûrement à des éléments concrets dans la représentation scénique du protagoniste. Est-ce pour autant que l'on doive parler de spectacle monstrueux et surtout non eschyléen? Un des lecteurs les plus avertis d'Eschyle, O. Taplin, postule que le visuel a une signification dramatique partout ailleurs chez Eschyle sauf dans le Prométbée ${ }^{14}$. Venant de celui qui a fait beaucoup pour réhabiliter le prétendu « spectaculaire » dans le théâtre eschyléen, ce jugement invite à refléchir sur ses fondements. Malgré son attitude très critique à l'égard de la Poétique, dans le cas présent, O. Taplin semble réactiver les catégories aristotéliciennes en joignant à la critique du visuel la thèse courante d'une tragédie pauvre en action et décousue, d'une exception dans la technique dramatique eschyléenne. Cependant, la critique de Taplin est des plus stimulantes, car elle résume les traits problématiques du Prométbée par l'alternative: «pas eschyléen »/ « caractéristique de la tragédie post-eschyléenne » ${ }^{15}$.

Prenant appui sur cette espèce de formalisation des écarts, je m'interroge : et si le Prométhée n'était pas une aberration dans le paysage du tragique, mais un moment de mutation que le « père de la tragédie » est en mesure d'entamer, comme tant d'autres innovations audacieuses? Des éléments troublants, comme l'inclusion de Zeus dans l'action - ne serait-ce que sur le mode ingénieux du silence - et le destin inhabituel du chour plaident en ce sens.

Au caractère novateur de la pièce s'ajoute un autre élément susceptible d'en perturber la réception : sa forte dominante «théologique ». En simple esquisse, je résume ce qui me semble «bloquer » l'accès à l'évaluation sereine de cette dimension majeure du tragique : la tendance plus générale de «laïciser d'entrée de jeu la cité ${ }^{16}$, renforcée - pas toujours consciemment - par l'implicite projet aristotélicien d'épurer la tragédie du religieux ${ }^{17}$. C'est ainsi qu'on peut, tout en étant connaisseur d'Eschyle, ouvrir un chapitre sur les dieux dans le théâtre eschyléen en affirmant : « in the eyes of Aristophanes, Aeschylus was a true believer... This is patent nonsense ${ }^{18}$.

Il me semble cependant que s'il y a une condition nécessaire pour que le Prométhée redevienne une tragédie à part entière, c'est celle d'accepter, avec Reinhardt, que dans cette pièce - peut-être plus que dans toute autre - Eschyle « prend les dieux à cœur ${ }^{19}$. Minimiser ce ressort fondamental du tragique réduit fatalement le Promé-

14 O. Taplin approche avec une grande prudence la question de l'authenticité, donnant dans l'appendice D de Stagecraft of Aeschylus les raisons qui le font pencher vers la thèse d'un Prométhée non eschyléen, tout en précisant que la question ne peut être tranchée. Pour une défense documentée et raisonnée de l'authenticité, voir S. SÄ̈D, Le sophiste et le lyran, Paris, 1985, p. 27-80.

15 "Prometbeus either sets a precedent for later tragic practice - or is itself a later tragedy" (TAPlin, o.c. [n. 7], p. 247) : Il serait trop long de reprendre ici le détail des passages commentés.

16 N. Loraux, La cilé divisée, Paris, 1997, p. 15.

17 De l'immense bibliographie trois lectures qui articulent très utilement l'argument: D. LANZA dans Calame, o.c. (n. 5); U. Eco, "D'Aristote à Poe", in B. Cassin, Nos Grecs et leurs modernes, Paris, 1992, p. 281-302; KLIMI, o.c. (n. 5).

18 Th. Rosenmerer, The Ant of Aescbylus, Berkeley, 1982, p. 259.

19 Rernhardi, o.c. (n. 13), p. 90. Je suis à l'aise dans cette formulation parce qu'elle n'engage pas l'idée d'une ouvre dramatique qui vise une théologie systématique, tout en postulant l'existence d'une sensibilité religieuse partagée, aussi bien de la part de l'auteur que du spectateur, élément indispensable en l'occurrence pour le fonctionnement du tragique. 
thée à un mélodrame sans profondeur; un " tract politique totalement partial ", et Zeus « à une allégorie de la tyrannie ${ }^{20}$.

Il ne s'agit pas pour autant de s'enfermer dans le schéma théologique universel du dieu souffrant, comme Farnell, qui considérait le Prométhée comme le problème le plus dur qu'un helléniste puisse affronter, avouant d'ailleurs, en 1933, l'avoir toujours évité. Farnell s'intéresse, bien évidemment, aux problèmes théologiques (majeurs !) que la pièce soulève. Kitto a ráison de lui répondre, l'année suivante déjà, que plus qu'un dieu souffrant, Prométhée est, pour Eschyle, un héros tragique : « Aeschylus was not anticipating the Christian Tragedy, but very surely and magnificently anticipating the Aristotelian $»^{21}$.

Mise au point indispensable, mais le cas est encore plus compliqué, face à la stratégie aristotelicienne - idéologiquement orientée - de "laïciser » la lecture de la tragédie. De ce point de vue, la lecture de Reinhardt est décisive car il donne à l'intrigue divine de la pièce toute sa portée tragique et scénique, allant même jusqu'à dire qu'Eschyle a l'intention de troubler ${ }^{22}$. Une telle attitude me semble « ré-animer » une pièce souvent perçue comme stagnante. Car Eschyle me semble soumettre la narration hésiodique à une espèce de temporalisation, faisant du supplice - et de la surprise qu'il représente pour Prométhée - l'ossature dramatique de la plus théocentrique des œuvres tragiques. À suive Solmsen, certains aspects de ce Zeus hésiodique - imposant dans la tradition grecque - ne cadrent pas avec celui d'Eschyle, qui admettrait mal l'idée d'un châtiment irrévocable et d'un bannissement éternel. Je dirais donc que, par cet espèce « d'arrêt sur image » qu'est le Titan au centre de la scène, le poète dialogue avec - ou « attaque »-Hésiode en agissant, en premier lieu, sur la temporalité ${ }^{23}$ : il suspend le temps hésiodique et ouvre un temps dramatique qui se focalise sur le supplice en faisant parler tout au long de la pièce le supplicié et ceux qui l'observent, créant ainsi un spectacle à l'intérieur du spectacle, pour faire ressortir progressivement la racine prométhéenne du genre tragique.

Le dramaturge est aux prises avec Hésiode; des impératifs idéologiques qui sont tributaires d'une conception religieuse du monde - mais qui ne se résument nullement à celle-ci - conduisent Eschyle à ce que Solmsen appelle subtilement « an extension of the hesiodic pattern », qui narre les choses « du point de vue de Zeus». Même si le scénique en soi n'est pas pris en compte, l'analyse de Solmsen ${ }^{24}$ me paraît féconde, car elle suggère une dynamique dans le rapport d'Eschyle à Hésiode, essentielle pour la compréhension de la pièce. Malgré le recours fréquent à la notion

20 Taplin, o.c. (n. 7), p. 469; Podiecki, o.c. (n. 11), p. 101 sq.

21 L.R. FARnell, "The Paradox of the Promethens Vinctus", JHS 53 (1933) p. 40-50; H.D.F. KitTo, "The Prometheus", JHS 54 (1934), p. 14-20.

22 Reinhardt, o.c. (n. 13), p. 90.

23 Sur le temps dans la tragédie, voir J. De Romlur, Le temps dans la tragédie grecque, Paris, 1971 (sur Eschyle, p. 57 sq.); D. LANZA, "Les temps de l'émotion tragique. Malaise et soulagement", Mètis 3 (1988), p. 15-39; Klım, o.c. (n. 5), p. 27 sq. pour une analyse de la conception normative du temps tragique chez Aristote,

24 Choisir entre Solmsen et Reinhardt à propos des questions théologiques n'est pas l'objet de cet article : il me semble qu'ils sont à relire attentivement à propos du Prométbée, car ils partagent l'idée d'un Eschyle "théologien » qui n'est toujours pas un acquis (F. Solmsen, Hesiod and Aeschylus, Ithaca, 1949) 
d'interpolation ${ }^{25}$, je retiens chez Solmsen la prise en compte tant de l'intertextualité que de l'hypothèse d'une dimension idéologique qui la motive : il me semble, en effet, qu'une approche globale du fait littéraire grec ne peut faire l'économie de cette double dynamique.

Transposition explosive d'un motif trop connu, le Prométhée ne laisse pas de place à une intrigue au sens classique du mot, ce qui nous rappelle l'impression de «vide dramatique », souvent reprochée à cette ouvre depuis Schmid ${ }^{26}$. Les particularités stylistiques de la pièce pourraient correspondre à la recherche d'une forme adéquate pour exprimer des nouveaux problèmes ${ }^{27}$ : odes chorales brèves, simplicité du style, fréquence exceptionnelle des répétitions ${ }^{28}$ - pourrait servir la logique d'un plaidoyer $^{29}$; il est important de convaincre, doublement. Car, si Eschyle a construit un Prométbée insolite, il faut que son protagoniste soit persuasif, qu'il prononce en même temps son propre plaidoyer et celui du poète. De ce point de vue, la nuance qu'apporte Garzya à l'expression «vide dramatique » me paraît tout à fait fondée : « drame pauvre en action théâtrale... riche en tension dramatique ${ }^{30}$.

Il me semble en effet, qu'on peut voir en cette pièce un extraordinaire « laboratoire » de l'émotion tragique et de ses origines : la récurrence et l'agencement des thèmes indiquent un intérêt poétique particulier pour la pitié et la terreur, chaînons importants de cette construction dramatique peu commode, dont l'univers est divisé entre ceux qui éprouvent et ceux qui n'éprouvent pas ces sentiments tragiques par excellence.

\section{Prométhée philanthrope}

Dès lors, la question se formule ainsi, inévitablement : comment une telle tragédie, qui se déroule essentiellement en milieu divin, pourrait-elle ressembler aux autres? On peut comparer Prométhée avec Cassandre ou Antigone ${ }^{31}$, mais il n'a d'autre fragilité que la douleur et l'orgueil, comme il le dit lui-même, tantôt en se vantant tantôt en se lamentant, ni la mort ni l'illisibilité de l'avenir ne le concernent (753). Dès lors, je crois que les émotions dans le Prométhée sont à redéfinir, car le protagoniste peut faire fi des limitations humaines fondamentales.

Par conséquent, la notion de l'action se modifie, car la pièce emprunte le thème mythique le plus adéquat pour faire une «archéologie » de l'émotion tragique, une élaboration de la notion de la compassion ${ }^{32}$ : choisir de mettre en scène le conflit et les passions dans le monde divin, c'est remonter à ce temps indécis où dieux et

25 Qui n'entame pas ce dynamisme. Voir, à propos de la cohérence du projet hésiodique, la mise au point de F. BLAISE, "Individualité d'un sens ou individu historique", in F. BLAISE, P. JUde'T de La Combe, Ph. Rousseau (éds), Le métier du mythe, Lille, 1996, p. 255-262.

26 Réfutation très pertinente des arguments de Schmid dans P. SÉchan, Le mythe de Prométhée, Paris, 1951, p. 59-64.

27 Voir pourtant les réserves de TAPLiN, o.c. (n. 7), p. 461-462.

28 SAÏD, o.c. (n. 14), p. 75-76.

29 ReINHARD', o.c. (n. 13), p. 86 sq.; LÉTOUBlon, l.c. (n. 12), p. 12-15.

30 A. Garzya, "Le tragique du Prométhée enchaîné d'Eschyle", Mnemosyne 18 (1965), p. 117.

31 Voir p. ex. C. HAInes, "Notes on the Parallelism between the Prometbeus Vinctus of Aeschylus and the Antigone of Sophocles", $C R 29$ (1915), p. 8-10.

32 Le terme étant une traduction « résumée » de l'abondance des mots composés de $\sigma u v-$ :

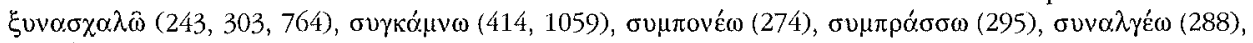
$\sigma v \mu \pi i ́ \tau v \omega(432)$, et non un anachronisme incontrôlé. 
hommes, encore proches, avaient en commun l'expérience de la souffrance. L'Iliade montre puissament cet état dans le dilemme de Zeus à propos de Sarpédon et surtout dans la douleur de Thétis, qui organise le thème iliadique ${ }^{33}$. Eschyle remonte vers cette ère confuse, vers un monde divin ébranlé par la matière par excellence du tragique, le patbos, et choisit pour cela un personnage thêâtal aux contours troublants.

En fixant son héros au milieu de la scène, Eschyle déploie les mécanismes même du spectacle, en mobilisant doublement la catégorie troublante que constitue l'humanité, acteur paradoxal, car quasi absent de la scène ${ }^{34}$, très présent dans le thème ${ }^{35}$, mais aussi sur les gradins, assistant à sa « préhistoire » mythique. Cet acteur paradoxal qu'est l'humanité joue un grand rôle dans le Prométhée, mineur sur le plan structurel, majeur sur le plan thématique, et c'est par le biais de la notion de " philanthropie » que nous l'aborderons brièvement pour nous aider d'appréhender par la suite les thèmes de la pitié et de la terreur.

Des ouvres que nous connaissons des trois tragiques, le Prométhée enchaîné est

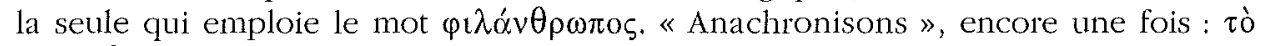
$\varphi \imath \lambda \alpha ́ v \theta \rho \omega \pi$ ov est un terme problématique dans la Poétique dénotant une certaine disposition en l'homme pour la réception du tragique, mot dont je résumerai les différentes interprétations comme suit: un sens moral de l'humain, un sentiment de l'humain, de communauté, Le débat autour de ce terme dans la Poétique - qu'Aristote associe étroitement à la pitié et la terreur - est hors de mon propos ${ }^{36}$; soit dit à titre anecdotique pour le moment que l'une des deux occurrences du mot dans la Poétique apparaît dans le chapitre où Aristote mentionne le Prométbée (1456a 21).

À propos de cette notion de « philanthropie », l'essentiel est dit dès le début de la pièce : selon les dires de Kratos, Prométhée a volé « le feu brillant d'où naissent tous les arts... pour l'offrir aux mottels. Pareille faute doit se payer aux dieux, - continue Kratos -, qu'il apprenne donc à chérir le règne de Zeus et à cesser les manières philanthropes » (7-11). Quelques vers plus loin, Hephaistos reprend, sur un autre ton, le thème de la philanthropie: "Voilà ce que tu as gagné avec tes manières philanthropes... », dit-il à Prométhée (28-30); ici pointe le thème de l'impossible réciprocité, qui rend l'acte de Prométhée illogique aux yeux de ses défenseurs, car c'est seulement eux qui se préoccupent de la $\mathrm{charis}^{37}$. Prométhée ne regrette rien, mais les autres dieux insistent sur l'assymétrie du rapport homme-dieu; ainsi Kratos : " en quoi les hommes peuvent-ils t'alléger de tes épreuves? (v. 85) », En fait, de Kratos à

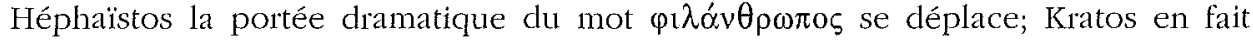
comme une menace au régime de Zeus, Héphaïstos met l'accent sur cette façon

33 À propos du rôle de Thétis dans l'Iliade, qui va bien au-delà de l'explicite, voir le beau livre de L. Slatkin, The Power of Thetis, Berkeley, 1991.

34 La distinction s'impose, me semble-t-il, entre l'humanité silencieuse et lo dont le rôle mérite un développement à part; j'aborde le personnage dans le cadre de l'étude sur l'éros, à paraître (voir supra, n. 6).

35 Tout en étant d'accord que « l'homme, l'humanité sont bien impliqués dans la tragédie, mais ils n'en épuisent nullement la matière ", Reinhardr, o.c. (n. 13), p. 92.

36 J. Moles, "Philanthropia in the Poetics", Phoenix 38 (1984), p. 325-335; C. SEgAl, "Catharsis, Audience, and Closure in Greek Tragedy", in SilK, o.c. (n. 7), p. 164-165. Voir aussi N. Loraux, "Ce que Les Perses ont peut-être appris aux Athéniens", Epokbé 3 (1993), p. 161.

37 A. PoDlecki, "Reciprocity in Prometheus Bound", GRBS 10 (1969), p. 287-292. 
d'agir sans espoir de contrepartie, qui condamne Prométhée à être fixé dans un état sans terpsis, réduit à la seule action des gémissements vains.

Le texte ne laisse aucun doute. Prométhée lui-même fait de la philanthropie la

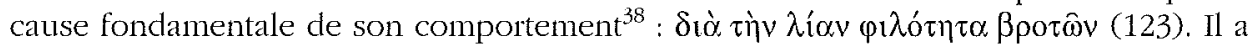
agi «par amour excessif des hommes». C'est que la philanthropie réintroduit le tragique là où il ne devait avoir aucune place, par définition : dans le monde divin, ou, pour être plus précis, là où il n'a de place que quand les humains s'en mêlent.

Dans cet aménagement eschyléen du thème de l'amour et de la haine dans l'histoire de l'univers, la philanthropie met en évidence une distinction importante. Entre Zeus et Prométhée, il y a une différence fondamentale du point de vue tragique : Zeus est étranger au sentiment de la pitié, en général ${ }^{39}$. Quant aux autres Olympiens, qu'ils sympathisent ou pas avec Prométhée, ils n'ont pas d'égards pour les mortels.

Il me semble que cette notion « d'amitié pour les hommes » affecte profondément le statut du public et son rapport à la pièce car l'œuvre tragique ici commentée n'a pas un hétos mortel, elle n'implique pas un genos illustre et maudit ${ }^{40}$, mais les hommes comme ensemble, comme « humanité » justement, et en fait un « acteur collectif » indirect impliqué dans le jeu cosmique entre amour et haine. Son absence de l'action est une option qui amplifie l'effet théâtral - le plus fort et le plus faible sont absents - en accentuant son impuissance dans l'affrontement des puissances divines, ce qui augmente le potentiel de sympathie en intensifiant l'action de la pitié et de la terreur. L'existence même de cette humanité collectivement nommée, dont Prométhée prononce le plaidoyer, permet une autre évaluation des énoncés divins contre le Titan, et rend la notion de la $\dot{\alpha} \mu \alpha \rho \tau i ́ \alpha$ prométhéenne presque caduque ${ }^{41}$. Par ce savant montage, la configuration eschyléenne des personnages représentant Zeus ne peut pas mobiliser la sympathie, l'adhésion de ce public - l'« humanité » - car Prométhée est son défenseur " ancestral ».

À lite ainsi la pièce, la notion de la philantropie apparaît comme une pièce importante de la construction du tragique, car c'est l'élément qui déclenche la dynamique de la terreur et de la pitié, en donnant à la pitié le statut du motif de renversement et en en faisant ultimement, par le moyen d'une thématisation récurrente, une force contre la terreur.

Le fait que ces forces, que nous avons appris à envisager comme les sentiments tragiques par excellence, sont des moteurs dans l'intrigue même nous conduit à remettre sur le métier la définition «tragédie simple» que nos présupposés aristotéliciens « plaquent » souvent sur le Prométhée en l'excluant du cortège des tragédies réussies : le schéma d'une complémentarité... « entre prépondérance du spectacle et simplicité de l'histoire » résume l'esprit de cette critique ${ }^{42}$. Or, il me semble que si la temporalisation eschyléenne du texte hésiodique consiste avant tout en une focalisation sur les émotions, le but est de mettre au coeur de la construction le supplice, point nodal du « désaccord» avec Hésiode, seul élément de surprise pour le Titan.

38 Sur l'importance de ce thème, voir Solmsen, o.c. (n. 24), p. 144 sq.

39) N. Loraux, La volx endenillée, Paris, 1999, p. 182-185.

40 Poétique, $1453 \mathrm{a}$

41 Griffith, o.c. (n. 11), aux vers 8-9; Kitto, l.c. (n. 21).

42 J'emprunte les mots de Dupont-Roc et Lallot qui se réfèrent bien évidemment au texte de la Poétique (p. 297). 
omniscient. À suivre donc les énoncés «tissés » dans la dynamique dramatique, c'est dans cette configuration qu'il faut chercher les ressorts de l'intrigue chers à Aristote. Ils existent dans le Prométhée, mais ils sont autrement agencés.

Car il y a un élément qui change tout et qui retrouve tout son poids quand on accorde à la pièce son envergure théologique : ce n'est pas l'agnoia du protagoniste qui sert de fondement aux effets dramatiques : Prométhée «savait tout d'avance », c'est même en connaissance de cause qu'il a commis son bamartia : " mais tout cela moi je le savais, voulue, voulue a été mon erreur - je ne veux point le renier... » (265266). Parler de stagnation revient à négliger le fait que le Titan connaît l'intrigue d'avance, il connaît même plus de choses que Zeus, le secret qu'il détient étant une menace porteuse de futurs renversements. Dans le temps présent, Prométhée formule la surprise en ces termes : « je ne pensais pas pourtant que de pareilles peines me devraient clessécher à jamais sur des cimes rocheuses et que j'aurais pour lot ce pic désert et solitaire » (268-270). Soulignons : ce n'est pas l'idée du châtiment en soi que Prométhée récuse - il dit lui-même, devant Hermès, qu'il n'y a rien de bizarre à ce qu'on soit châtié par l'ennemi (1041-1042), il en tire presque fierté. Or, c'est justement cet élément de surprise qui est la source la plus puissante de pitié, comme si la parole eschyléenne devait constituer en injustice la version hésiodique de ce thème.

Néanmoins, il est très hâtif d'exclure entièrement la metabolê du plan dramatique du Prométhée, même s'il est vrai que le renversement - projeté vers le passé ou le futur - est surtout récit et pas action ${ }^{43}$ dans la pièce. Car, au sein de cette dynamique des émotions, c'est l'oiktos qui agit comme motif de renversement, doublement: dans ce qui est le passé dans la pièce, la pitié à l'égard des hommes était à l'origine de la chute de Prométhée, mais, dans le temps présent, la pitié et la terreur sont le motif principal de la disparition du choeur des Océanides, son fídèle auditoire interne, qui s'engouffre avec lui à la fin de la pièce ${ }^{44}$. L'importance de ce renversement a été trop minimisée à mon avis; or, en tant qu'aboutissement du corps à corps des mots ${ }^{45}$ qu'est l'affrontement entre terreur et pitié tout au long de la pièce, c'est la pitié qui dominera dans le registre de la persuasion, comme si la vision d'Eschyle devait l'emporter sur' celle d'Hésiode.

Ce sont les vers 238-239 qui énoncent le plus clairement l'imbrication de la pitié dans l'action : Prométhée a seul osé sauver les hommes du verdict de Zeus, dit il, en mettant avant tout le sentiment de pitié pour les hommes : "j'ai osé ... et c'est là pourquoi je ploie sous de telles douleurs, cruelles à subir, pitoyables à voir. Ayant dans la pitié privilégié les mortels, je me suis vu refuser la pitié et voilà comment, sans clémence, je suis ici traité, spectacle funeste au renom de Zeus » (235-241). Le verbe غ̇ंó $\lambda \mu \eta \sigma \alpha$ annonce déjà la crise : la démesure de l'acte tragique chez Eschyle trouve souvent son origine dans l'audace, signe inquiétant de dépassement des limites,

43 Sur cette distinction importante, voir T. Todozov, Poétique de la prose, Paris, 1978, p. 27 sq. Voir v. $907 \mathrm{sq}$. pour le renversement dans le futur.

¿4́ A propos de cette « disparition » et des réserves de certains érudits, consulter Taplin, o.C. (n. 5), p. 272; un résumé de la discussion est clonné par D.J. Cronacher, Aeschyllis Prometheus Bound. A Literany Commentay', Toronto, 1980, p. 175 sq. Même si on ne partage pas cette vue, il est clair que le chœeur adhère à la fin à la position de Prométhée. Sur les questions concernant la mise en scène, voir N.G.L. Hammon, "More on the conditions of production to the death of Aeschylus", GRBS 13 (1987), p. 5-33.

45 J'emprunte l'expression à Nicole Loraux, "La métaphore sans métaphore", Europe (janvierfévrier 1999), p. 249. 
fréquemment utilisé dans l'Orestie ${ }^{46}$. Vient après la répétition dense des notions relatives à la pitié, pour montrer un Prométhée qui s'implique dans le cercle tragique de la pitié et de la terreur ${ }^{47}$, en premier spectateur compatissant de la condition humaine.

À ces mots de Prométhée qui situent son action sur le plan des émotions, le chœur répond en reprenant le thème du caractère insupportable du supplice dont la vue fait souffrir le coeur, et on ne manquera pas de remarquer la critique indirecte de ceux qui sont «sans pitié » : «Il aurait un cœur fait de roc ou de fer, Prométhée, celui qui ne s'indignerait avec toi de tes peines. Je n'eusse pas, pour moi, souhaité voir un tel spectacle, et à le voir mon cœur douloureusement s'émeut » (242-245). A quoi Prométhée répond : «Oui, j'offre à des amis une vue pitoyable » (246).

\section{La métaphore sans métaphore}

Sans le vouloir, car il arrive sur scène entraîné par ce ce qu'on peut appeler un effet scénique sonore (v. 133-134), le chœur sera pris dans un agôn ${ }^{48}$ dont un des interlocuteurs principaux, Zeus, est absent, sa parole étant relayée par l'acte spectaculaire de clouer, façon de dire, dans les Suppliantes, la décision ferme ${ }^{49}$. Dans cet affrontement entre les puissances de mise en ordre du monde, les Océanides assument un rôle peu habituel, qui met en difficulté la distinction entre personnage et chour dans la tragédie ${ }^{50}$.

Face à Prométhée, le chœur est l'élément par excellence qui relaie la thématique des émotions. Jaeger dans sa Paideia y a vu la préfiguration du modèle aristotélicien du tragique, profonde conscience des effets du drame, dont la catharsis s'opère par la décision de partager le sort du Titan ${ }^{51}$. Reinhardt nuance substantiellement cette interprétation : ce n'est pas du pathos en soi qu'il s'agit ici; une telle lecture « séculariserait » l'enjeu dramatique de cette intrigue divine ${ }^{52}$. Pris d'effroi devant le spectacle

46 Sur l'audace et la crainte dans le prométbée, voir SÄ̈D, o.c. (n. 14), p. 319-325.

47 Voici le contexte de ce passage, très important dans une pièce où la terreur et la pitié sont constamment évoquées :

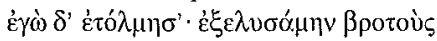

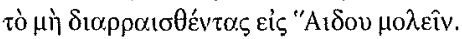

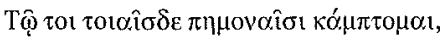

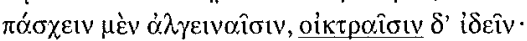

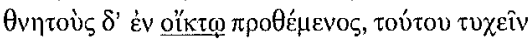

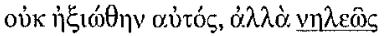

$\hat{\omega} \delta$ '

Le vers 267 introduit idée d'un «échange» aussi sinistre que concret : pour avoir aidé les hommes, il subira un des aspects les plus durs de la condition humaine, sans la contrepartie d'une

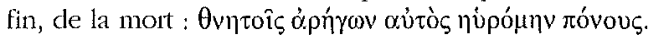

48 Reinhardt, o.c. (n. 13), p. 87. L'expression de S. Goldhill « agon of attitudes » à propos de la tragédie convient tout à fait au Prométbée; "Collectivity and otherness - The Authority of the Tragic Chorus: response to Gould", in Silk (éd), o.c. (n. 7), p. 252.

49 Dans les Suppliantes, c'est ainsi qu'on exprime la stabilité d'une décision, un clou

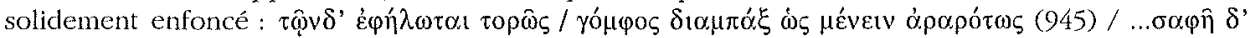

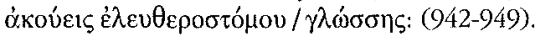

50 Comme le dit Scott dans un article important, "The development of the Chorus in the Prometheus Bound", TAPbA 117 (1987), p. 96 : "Chorus is a character in the Promethets Bound..."

51 W. JAEger, Paideia (trad. fr), Paris, 1964, p. 308-313.

52 Reinhardt, o.c. (n. 13), p. 84-85. Voir aussi Létoublon, l.c. (n. 12), p. 25. 
d'une violence extrême, le chour essaie de se tenir à l'extérieur du tragique, tant que la dynamique dramatique ne l'oblige pas à sortir du rôle de spectateur : tout en compatissant, les Océanides critiquent Prométhée de ne pas connaître le sentiment de la peur, car c'est entre ces deux excès - manque de pitié et manque de peur - que la démesure s'opère dans une pièce où les sentiments régulateurs font défaut : " Mais je frémis quand ici je te contemple, déchiré de milles maux. Sans crainte de Zeus, ta volonté indocile a trop d'égards pour les mortels, Prométhée ${ }^{53} »$.

Peu à peu les éléments qui articulent l'expérience du chœur se mettent en place: dans le deuxième stasimon, l'ode qui précède l'entrée d'Io, le chœur dit - croit - avoir

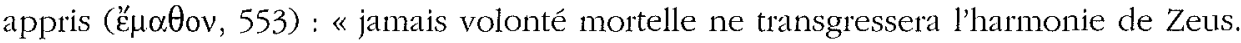
J'ai appris cela en contemplant ton malheur Prométhée. » Mais le rapport de la souffrance au savoir n'est pas le même que dans l'Agamemnon, et le chœur sera finalement confronté à un autre type d'« apprentissage ». C'est le thème de l'humanité qui sera décisif à ce propos : le spectacle d'Io rendra visibles l'injustice de Zeus à l'égard de l'humanité et le contre-exemple de l'attitude " philanthrope » de Prométhée : la cruauté du dieu suprême a déformé Io $^{54}$, en la privant de sa semblance humaine. C'est par ce renvoi incessant du thématique au visuel que le chœur va atteindre le but de ce que nous appelons la leçon tragique. Au contact de la figure de la prescience qui raconte l'histoire des dieux et des hommes, à la vue de l'audace qui défie la terreur et la souffrance, le chœur semble vivre une expérience qu'on pourrait qualifier d'initiatique, puisqu'il change d'état et de comportement peu avant la fin de la pièce ${ }^{55}$.

Il y a des signes annonciateurs de cette transformation dès le début de la pièce. On les lira ainsi surtout si l'on pense, avec Nicole Loraux, que le sens aristotélicien de la métaphore ne recouvre pas "le travail des mots sur les choses » dans le langage eschyléen, du moment où les images qui paraissent métaphoriques sont vouées à se réaliser ${ }^{56}$. À commencer par l'effet sonore qui, au début de la pièce, « appelle » les Océanides sur scène: "Car l'écho des chocs du fer a traversé le fond de mon antre, et mis en éclat la pudeur qui pesait sur mon visage, et je me suis lancée, pieds nus, sur ce char ailé » (133-135).

Il vaut mieux « se tenir à l'étrangeté du mot-à-mot » : par un écho « cosmique », la violence des coups du marteau qui clouent Prométhée, atteignent non seulement le «fond » de la demeure des nymphes ${ }^{57}$, mais aussi leur façon d'être, car ils « heurtent» la pudeur, réveillant ainsi l'audace qui les lance hors de leur univers confiné. Or, le « déracinement» d'un chœur tragique n'est jamais anodin ${ }^{58}$ : si les nymphes expriment l'effet de la peur dans leur corps par ce même mot qui désigne la transpersion des membres de Prométhée par les liens ${ }^{59}$, c'est que leur sort devient prisonnier du sien. En l'occurence, l'indistinction entre le propre et le figuré anticipe l'implication de ces créatures timides dans une expérience peu commune, les rendant témoins privilégiés d'un chamboulement cosmique.

53 541-543, voir aussi 950-961.

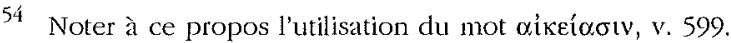

55 Griffith, o.c. (n. 11), au vers 162; Scott, l.c. (n. 50) : "One must speak of conversion, 》

56 Loraux, "La métaphore sans métaphore", l.c. (n. 45), p. 244.

57 Voir S. Des Bouvrie, "Aeskhulos, Prometheus. An anthropological approach", Métis 8 (1993),

p. 202-216, spéc. p. 202-203; A. Ballabriga, Les fictions d'Homère, Paris, 1998, p. 128 sq.

58 Voir GoldhilL, l.c. (n. 48), p. 245.

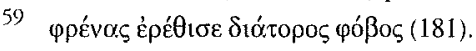


Apparaît ainsi une structure tragique plus complexe qu'il n'y paraît, où la distinction entre les catégories du chœur et du personnage devient plus trouble. Le rapprochement entre Prométhée et les nymphes, qui s'opère de façon très progressive, s'exprime dans la forme tragique par excellence qu'est la lamentation. Car le choix poétique des Océanides pour accompagner Prométhée dans son épreuve et répondre à un thrène dont il est le sinistre coryphée n'est pas fortuit : plus loin dans la pièce, on apprendra qu'elles sont les sours d'Hésioné, l'épouse de Prométhée. Bien à leur place dans le contexte du deuil, elles accompagnent une souffrance sans fin, sans possibilité de soulagement par la mort.

\section{La force du thrène}

Le thrène sur la scène tragique a été " entendu » par Nicole Loraux comme le lieu d'expression par excellence de la spécificité tragique, au sein d' une cité qui a accordé une attention particulière à la maîtrise des émotions dans la vie civique. C'est dans sa dimension "contestataire " ou «alternative » que la "voix endeuillée » de la tragédie ${ }^{60}$ puise sa véhémence : l'humain est pour les tragiques « la mesure de toute chose », y compris de la chose politique que le thrène théâtral « interpelle », comme le Titan souffrant défie Zeus.

« Je viens juste de terminer la lamentation sur mes peines », dit Prométhée, au début de l'épisode d'Io (au vers 615), qualifiant ainsi toute sa prestation dramatique précedente. En effet, le temps présent du Prométbée est scandé par la lamentation, élément particulièrement actif dans la dialectique de la pièce, mais qui ne suffit pas en lui-même pour produire du tragique. Le principal effet dramatique dans le Prométhée tient, me semble-t-il, à la mise en scène " de la figure tragique par excellence * qu'est l'oxymore ${ }^{61}$ : sous forme d'extrême écart entre opsis et lexis, entre corps souffrant - « visualisé » dans le discours du protagoniste et ses « spectateurs » internes - et langage audacieux du supplicié ${ }^{62}$. Fidèle à sa « mission » théâtrale, le chœur exprime cette tension insupportable dès la parodos, après un discours particulièrement véhément de Prométhée à l'égard de Zeus : «l'effroi des plus dures menaces ne me fera pas révéler ce secret, à moins qu'il n'ait d'abord déserré les liens farouches et consenti à payer le prix dû à pareil outràge » (173-177), à quoi le chour répond : « Tu es fier, la détresse amère ne te fait rien lâcher; ta bouche est trop libre. Une frayeur perçante excite mon esprit »(178-181) ${ }^{63}$. Le phobos qui transperce naît de cet effet de contradiction entre liens et paroles libres, contradiction qui atteint à son tour l'esprit du chour...

À la fin de la pièce (944 sq.), l'agôn entre Prométhée et l'émissaire de Zeus, Hermès, constitue la climax de cette tension entre parole libre et liens implacables: pour accentuer encore l'écart entre ces deux éléments, le poète dote le Titan d'une

60 À part le livre qui porte le même nom, voir aussi Mères en Deuil, Paris, 1990. Ces travaux de N. Loraux permettent de ne pas lire la pièce comme un mélodrame (contra, voir B. Marzullo, I sofismi di Prometeo, Florence, 1993). Voir également l'article lumineux de Charles Segal, "Lament and Closure in Antigone", in Sophocles' Tragic World, Cambridge, Mass, 1995, p. 119-137, avec des références à ses autres travaux sur le même sujet.

61 Loraux, o.c. (n. 39), p. 96.

62 Une longue caractérisation du discours de Prométhée est donnée par Okéanos (309-327). Sur Prométbée comme pièce paradoxale où les liens et la liberté ne sont pas antinomiques, voir LÉTOUBLon, o.c. (n. 12), p. 36.

63 Cette traduction est de M. Gondicas, P. Judet de La Combe, légèrement modifiée. 
polyvalence dramatique exceptionnelle. La preuve en est donnée dès sa première prestation dramatique : l'extraordinaire variété métrique de son premier monologuemonodie (88-127) est unique dans le langage tragique, un passage alterné des parties chantées et non chantées: "Helpless self-pity, majestic confidence, and terror all surface within that single speech ${ }^{64}$. Sa première intervention est un mélange des formes mais aussi des états émotionnels.

On pourrait dire que Prométhée pense comme un dieu et souffre comme un homme ${ }^{65}$, «bloqué » qu'il est dans un état intermédiaire que les autres dieux ont

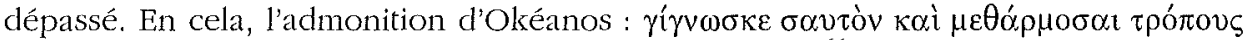
v'́ouৎ (309-310) - au-delà de ses échos « delphiques » ${ }^{66}$, n'a pas le même sens que pour un destinataire humain. Dans la représentation eschyléenne du monde indécis des nouveaux souverains divins, Prométhée doit connaître ses limites vers le haut mais aussi vers le bas, son $\sigma \varepsilon \hat{\varepsilon} \beta \alpha_{\zeta}$ à l'égard des mortels (543) est symétrique à son arrogance à l'égard de Zeus. Dans ce monde, le mélange est encore de mise, ce que dit d'une façon magnifiquement elliptique Prométhée dans sa monodie : « Ah quel bruit, quelle odeur obscure a volé jusqu'à moi ? parfum divin ? humain ? ou

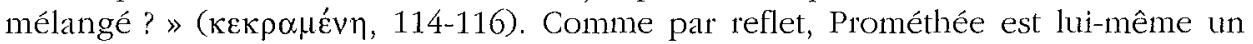
mélange d'états différents, être emblématique de cette période où la loi de la séparation définitive des éléments n'est pas encore instaurée. Il n'est sans doute pas indifférent à ce propos que le langage même de son premier monologue-monodie soit aussi « mélangé ».

Si le langage de ce Prométhée théâtral, être double sur le plan dramatique, est troublant, c'est surtout parce que cet oxymore formé par le corps terriblement souffrant et le langage démesurément libre se reproduit à l'intérieur même de son discours qui consiste en une interférence constante entre thrène et «auto-affimation ». Je ne m'attarderai pas ici sur cette dernière notion, - interprétation du mot récurrent $\left.\alpha \theta_{\alpha} \alpha_{\delta} \eta\right\rceil \zeta$ - qui résume chez Reinhardt ${ }^{67}$ l'éloge que Prométhée fait de lui. Car Prométhée ne peut obtenir la pitié qu'en faisant son propre éloge qui interfère constamment avec la lamentation ${ }^{68}$. Prononcés par le protagoniste concemé, lamentation et louange se confondent pour produire une forme tragique de $\alpha \dot{v} \theta \alpha \delta$ í $\alpha$, terrain de tension fondamental dans le Prométhée, car dans le temps présent de la pièce - où l'action est subordonnée aux logoi - ce n'est pas l'amitié pour les hommes $(\varphi \imath \lambda \alpha v \theta \rho \omega \pi i \alpha)$ qui constitue la transgression ( $\dot{\alpha} \mu \alpha \rho \tau i \alpha)$, mais le langage même de Prométhée, qui est qualifié d'insolent par ses adversaires ${ }^{69}$.

Dans le long échange entre Prométhée et le chœur, qui encadre le récit des bienfaits à l'humanité, une égale attention est accorclée à l'action et à l'émotion qui accompagne le récit de celle-ci. Ce dialogue se déroule sous le signe des effets douloureux du discours qui rapporte des événements malheureux : «En parler déjà

64 J. Herington, Aescbyllis, Yale University Press, 1968, p. 168-169; voir aussi Griffith, o.c. (n. 11), ad loc.

65 Voir sur la vulnérabilité des dieux, N. Loraux, "Le corps vulnérable d'Arès", Temps de la refléxion, 7 (1986), p. 335-354.

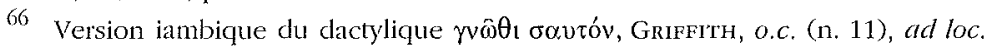

67 GrifFith, o.c. (n. 11), p. 52-60, aux vers 64-65.

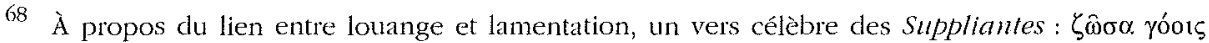
$\mu \varepsilon \tau \mu$ î̀ (116), voir LoRaux, o.c. (n. 39), p. 90.

69 Léroublon, l.c. (n. 12), p. 22-23; Kitto, l.c. (n. 21), discute la notion de la bamartia dans le Prométbée. 
est douloureux, mais me taire aussi m'est une douleur, de tous côtés le mauvais destin » (197-198); et se termine, dans une sorte de "ting composition » sur un topos tragique, indice de la fonction « générique » de la lamentation, de la pitié même, dans la tragédie : «cédez à la persuasion, cédez, compatissez à qui souffre à cette heure; de la même manière dans son errance le malheur se pose tantôt sur les uns tantôt sur les autres » (274-276).

La lamentation est donc un objet de discussion important dans la pièce, elle est différemment évaluée par les personnages. Si nous avons vu Kratos, « refuser d'une façon ou d'une autre les valeurs de l'univers tragique» par contre, le langage de Prométhée est celui d'un ardent défenseur du patbos, appliquant la loi selon laquelle les personnages tragiques souvent « usent de la lamentation à leur propre usage et à leur propre sujet $»^{70}$. C'est ainsi que les autres décrivent l'état de Prométhée : il en est réduit à prononcer « des plaintes sans fin, des lamentations vaines » (34-35).

Des mots vains, incapables d'infléchir le destin, qui se confond dans ce cas avec

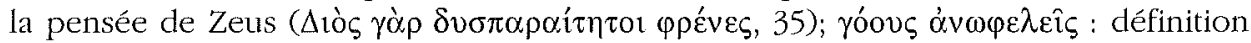
fondamentale de la lamentation du point de vue divin. C'est dans le même sens que se prononce Kratos quand Héphä̈stos le qualifie de « toujours sans pitié et plein de dureté », à l'image de son maître; à cela Kratos lui répond: "se lamenter sur Prométhée ne le guérira pas; ne peine pas pour quelque chose qui ne sert à rien » $(42-44)^{71}$. Radicalement exempt du patbos, le langage représentant la pensée de Zeus est souvent mis en opposition avec le discours de Prométhée, aussi explicitement qu'elliptiquement, par exemple, aux vers 979-980 : Pr. « Hélas », Herm. «Voilà un mot qu'ignore Zeus ». Car les nouveaux dieux, comme dit Prométhée, « croient habiter une citadelle inaccessible au deuil » (955-956).

La lamentation emprunte ici la figure du langage de l'impuissance face à l'irrévocable; aux yeux des pragmatiques, l'ingrédient principal du genre tragique est inefficace. Ainsi s'opère, magistralement, l'articulation délicate et toujours précaire entre affect et politique, faisant du Prométhée une haute manifestation de « ... la manière dont la tragédie, jouant dans l'oxymoron des incompatibilités posées par le discours civique, définit sa spécificité par le mélange de ce que le discours politique, quant à lui, oppose ${ }^{72}$. Les hasards de la transmission ${ }^{73}$ nous privent de connaître le sort réservé à ce défi que Prométhée « philanthrope » lance au Pouvoir absolu. Tout porte à croire pourtant qu'ici se trouve articulé plus pleinement le conflit entre idéologie civique et langage tragique, la cité des dieux étant divisée selon ce mode de partage.

Ce mélange de registres est très évident dans la joute oratoire entre Hermès et Prométhée qui est un agôn, pas un débat, comme le dit bien Reinhardt ${ }^{74}$. C'est le

70 Loraux, o.c. (n. 39), p. 88-90.

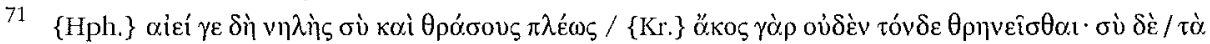

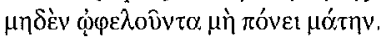

72 Loraux, o.c. (n. 39), p. 101. Voir aussi C. MEIER, De la tragédie grecque comme art politique, Paris, 1991, p. 174 sq., pour une autre lecture du politique dans le Prométhée.

73 Y a-t-il eu évolution, réconciliation, compromis («pacte » même, comme l'a proposé J. Bollack, lors d'une communication au colloque Corhali 1999)? Il n'y a même pas d'accord sur l'existence ou la structure trilogique que formeraient l'Enchấné, le Porte-fét et le Déliuré. Voir SÉchan, o.c. (n. 26), p. 49-80; G. THOMSON, Aeschylus and Athens (1941), 1973, p. 297 sq.; REINHARDT, o.c. (n. 13), 78-94 et 234-289; Des Bouvrie, l.c. (n. 57).

74. Sur le choix d'Hermès comme interlocuteur de Prométhée, il y a beaucoup à dire : voir Reinhardt, o.c. (n. 13), p. 86-87. Voir les réserves de J. Duchemin, L'áyóv dans la tragédie grecque, 
caractère agonistique des logoi qui aura donné à la pitié cette force renversante et qui accordera à Prométhée une victoire à la hauteur de son supplice : la force de sa parole a ébranlé les catégories « choeur-acteur», offrant une illustration complexe de la dynamique autoréferentielle selon laquelle "le récit mythique dans la tragédie dramatise avant tout le mythe de sa propre écriture ${ }^{75}$. Dans cette première pièce de la trilogie, la violence, même radicale, échoue dans son rôle de génératrice de persua$\operatorname{sion}^{76}$, et c'est la pitié qui l'emporte : «Ne vous lamentez pas sur mes souffrances présentes, mettez pied à terre, pour écouter le sort qui approche, vous saurez tout ainsi, d'un bout à l'autre » (271-273) 77 .

«Posez le pied sur terre », ainsi parle Prométhée aux Océanides, au moment où il les installe dans leur rôle - fatal - de chœur. Dans l'Agamemnon, Clytemnestre arrête Égisthe quand il ordonne la mort du chour ( $v$. 1650) car, comme le relève Nicole Loraux $^{78}$, « on ne tue pas le chour ». Pourtant, dans le Prométbée, Zeus le fait disparaître, en signe ultime de mépris pour le langage tragique... Dès lors, comment s'empêcher de penser qu'il fallait la stature d'un Eschyle pour représenter ce Prométhée aussi intrinséquement indispensable pour Zeus que Métis dans la Théogonie, et pour révéler une divinité suprême jeune, encore inaccomplie, loin de celle des Suppliantes, dont l'« arme n'est point la violence et qui règne sans peine » (v. 98).

Craignant la « contamination » par cet état bien humain qu'est la souffrance, les dieux grecs ont voulu mettre fin à l'état originel de proximité avec les mortels. Puisant dans ce topos du discours grec sur les origines, le Prométhée d'Eschyle met en scène le deuil en tant qu'opérateur de distinction entre dieux et hommes, et fait du Titan supplicié la figure emblématique de la souffrance tragique, évoquant avec force la lecture ultime que Nicole Loraux a faite de la tragédie grecque : «... par l'évocation du deuil, contre la prescription de l'oubli par la cité, [la tragédie] bouleversera le spectateur, elle l'incitera à dépasser son appartenance à la communauté civique pour saisir son appartenance, plus essentielle encore, à la race des mortels. Car telle est bien à jamais le dernier mot de ce que chante, au spectateur plus qu'au citoyen, la voix endeuillée de la tragédie ${ }^{79}$ (p. 137).

Ioanna PAPAdopoulou-BeLmeHd

Université Libre de Bruxelles

Paris, 1968, p. 49. Sur l'interférence de la pensée juridique avec la tragédie, J.-P. Vernant, P. VidalNaquer', Wythe et tragédie en Grèce ancienne, Paris, 1972.

75 P. Pucci, "Écriture tragique et récit mythique", Europe (janv.-fév. 1999), p. 213.

76 « La peitbô chez Eschyle est toujours le fruit de la violence», dit P. Pucci, Enigma, Segreto,

Oracolo, Roma, 1996, p. 61 et 107 sq.

77 Voir aussi 637-639.

78 L.c. (n. 2), p. 171-172.

79 Loraux, o.c. (n. 39), p. 137. 